\title{
PRODUCTION OF ULTRA-SHORT, HIGH CHARGE, LOW EMITTANCE ELECTRON BUNCHES USING A 1 GV/M DC GUN.
}

\author{
M.J. de Loos, S.B. van der Geer, Pulsar Physics \\ J.I.M. Botman, O.J. Luiten, M.J. van der Wiel, TU-Eindhoven
}

\begin{abstract}
Advanced acceleration schemes, for example those based on wake fields of laser pulses traveling through plasma, require the injection of very high quality relativistic femtosecond electron bunches. Such bunches can be produced by a photoexcited RF gun followed by longitudinal bunch compression. Currently we are investigating a different pre-acceleration scheme, which avoids the necessity of magnetic compression and the associated potential emittance growth due to coherent synchrotron radiation.

Instead of an RF cavity, we propose $1 \mathrm{GV} / \mathrm{m}$ DC acceleration of laser excited electrons across a $2 \mathrm{~mm}$ gap, following recent developments at Brookhaven Nat. Lab. [1]. The gun is powered by a $2 \mathrm{MV}, 1 \mathrm{~ns}$ pulse. Simulation results using the General Particle Tracer (GPT) code show that with the DC gun scheme a $100 \mathrm{pC}$ bunch can be accelerated to $2 \mathrm{MeV}$ with a final bunch length of $70 \mathrm{fs}$ and an emittance well below $1 \pi \mathrm{mm}$ mrad.
\end{abstract}

\section{INTRODUCTION}

At the Eindhoven University of Technology a new project has been started with the goal to do experiments with coherent transition radiation and plasma acceleration. For these experiments a new electron gun with very strict requirements is being designed. The design goals are a bunch charge of $100 \mathrm{pC}$ with a bunch length of $100 \mathrm{fs}$. Furthermore an emittance below $1 \pi \mathrm{mm}$ mrad and an energy of $10 \mathrm{MeV}$ is required.

To meet these requirements two possible routes can be followed. The first is to use an RF photoinjector and perform bunch compression. The problem with this method is that bunch compression systems based on for example chicanes cause the emission of coherent synchrotron radiation and this has undesirable effects on the bunch length and energy spread. At this point it is not clear whether the listed requirements can be met using this method.

An alternative method is to eliminate the bunch compressor by generating a short bunch and keeping it short. Initial simulations show that for this method an acceleration field of at least $500 \mathrm{MV} / \mathrm{m}$ is required until the beam is relativistic. Lower acceleration gradients produce longer bunches. Such fields can be achieved by means of a pulsed power supply, producing a DC voltage of $2 \mathrm{MV}$ during $1 \mathrm{~ns}$. A power supply of these specifications is presently being developed for this project at the Efremov Institute, St. Petersburg. When this pulse is applied across a diode with a gap of $2 \mathrm{~mm}$ a $1 \mathrm{GV} / \mathrm{m}$ field is achieved. Fields of this magnitude can be maintained without breakdown for the duration of $1 \mathrm{~ns}$ [1]. The drawback of this method is that a second acceleration stage is needed to increase the beam energy from $2 \mathrm{MeV}$ to $10 \mathrm{MeV}$.

From the simulation results the method based on the diode followed by an RF booster looks very promising. Therefore we have chosen to follow this path. In this paper we will present the simulation results for the diode and give a brief description of the used simulation package.

\section{THE DIODE SET-UP}

The diode set-up consists of a hollow spherical copper cathode and a flat anode with a circular aperture, schematically shown in Figure 1. The field in the diode is produced by a power supply generating a pulse of 2$2.5 \mathrm{MV}$ during $1 \mathrm{~ns}$. When this negative pulse is applied across the gap, acceleration fields of $1 \mathrm{GV} / \mathrm{m}$ are attainable. Because of the extremely high gradient and because no additional focusing is needed, this device could be much better suited for ultra-short bunch generation than a conventional photo-cathode.

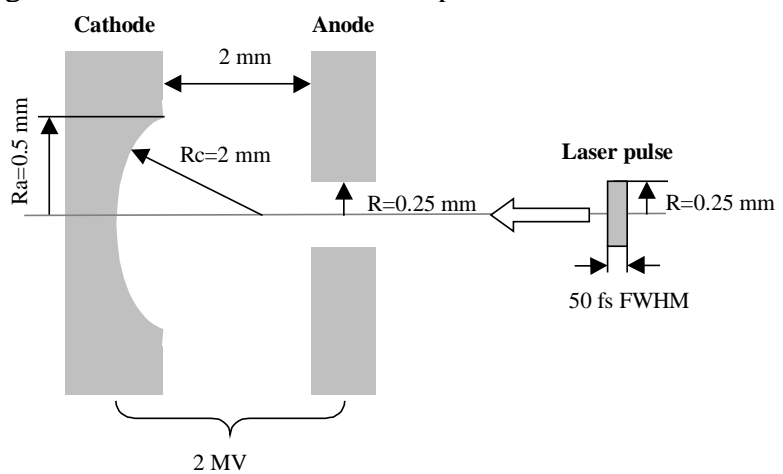

Figure 1: Schematic of the diode (not to scale).

The cathode is curved to produce a transverse focusing field, thus eliminating the need for external focusing. The opening in the anode is kept as small as possible to prevent the field from leaking out of the gun and thereby lowering the acceleration gradient. The set-up parameters are listed in Table A.

The laser used to photoexcite electrons on the cathode surface is injected on-axis and limited in radial size by the 
anode aperture. The very high electric field in the diode results in a considerable lowering of the surface work function due to the Schottky effect. This means that the pulses from the Ti:Sapphire laser, operating at $810 \mathrm{~nm}$, will only need to be frequency-doubled to extract electrons from the copper surface by single photon emission. The minimum pulse length of the laser we plan to use for the experiments is $50 \mathrm{fs}$ FWHM for a gaussian temporal profile.

Table A: Set-up parameters.

\begin{tabular}{ll}
\hline Parameter & Value \\
\hline Input voltage & $2 \mathrm{MV}$ \\
Electric field & $1 \mathrm{GV} / \mathrm{m}$ \\
Cathode curvature radius & $2.0 \mathrm{~mm}$ \\
Cathode aperture radius & $0.5 \mathrm{~mm}$ \\
Gap length & $2.0 \mathrm{~mm}$ \\
Anode length & $1.5 \mathrm{~mm}$ \\
Anode aperture radius & $0.25 \mathrm{~mm}$ \\
Laser radius & $0.25 \mathrm{~mm}$ \\
Initial emittance & $0.12 \pi \mathrm{mm} \mathrm{mrad}$ \\
Bunch charge & $100 \mathrm{pC}$ \\
Laser pulse length & $50 \mathrm{fs} \mathrm{FWHM}$ \\
\hline
\end{tabular}

\section{THE GENERAL PARTICLE TRACER}

The simulations for the design of the diode were performed using the General Particle Tracer (GPT) simulation package $[2,3]$. GPT is a $3 \mathrm{D}$ particle tracking code developed for the design of accelerators and beam lines. The differential equations for the particle trajectories are solved using a fifth order embedded Runge-Kutta method. GPT includes many standard elements representing beam line components and custom elements can easily be added by the user. It also incorporates a number of different space-charge models. Furthermore it includes a Windows 95/NT user interface complete with data analysis and graphics. The code is written in $\mathrm{C} / \mathrm{C}++$ and UNIX versions are also available. For the simulations presented in this paper, GPT was used in combination with the POISSON [4] set of codes to import the electric field-map of the diode.

\section{$3.12 \mathrm{D}$ space-charge model}

For the diode simulations GPT's 2D space-charge model was used, instead of the standard 3D point-to-point model. The 2D space-charge model can be used to calculate the effect of self-fields in axially symmetric beams by representing every particle as a homogeneously charged circle. Because every particle represents a complete ring, the amount of particles needed to obtain correct statistics is less than in $3 \mathrm{D}$ point-to-point calculations.

The equations for a homogeneously charged circle with radius $R$ and charge $Q$ in its rest frame are given by [5]:

$$
\begin{aligned}
& E_{r}^{\prime}=\frac{Q}{4 \pi^{2} \varepsilon_{0} r \sqrt{d^{2}+4 R r}}\left(K(\alpha)-\frac{R^{2}-r^{2}+z^{2}}{d^{2}} E(\alpha)\right) \\
& E_{z}^{\prime}=\frac{Q z E(\alpha)}{2 \pi^{2} \varepsilon_{0} r \sqrt{d^{2}+4 R r}}
\end{aligned}
$$

where $d^{2}=(R-r)^{2}+z^{2}, \alpha=4 R r /\left(d^{2}+4 R r\right)$ and $K$ and $E$ are elliptic integrals. Transforming this field back to the lab frame and adding the contributions of all particles $j$ yields:

$$
\begin{array}{ll}
E_{x}=\sum_{j \neq i} \gamma_{j} E_{x, j}^{,} & B_{x}=\sum_{j \neq i}-\gamma_{j} \beta_{z, j} E_{y, j}^{,} / c \\
E_{y}=\sum_{j \neq i} \gamma_{j} E_{y, j} & B_{y}=\sum_{j \neq i}+\gamma_{j} \beta_{z, j} E_{x, j}^{\prime} / c \\
E_{z}=\sum_{j \neq i} E_{z, j}^{,} & B_{z}=0
\end{array}
$$

This cylindrical symmetric space-charge model is fully relativistic and has a fast convergence. However there is a small error in the transverse electric field near the axis due to the finite number of rings included in the simulation. This is due to the neglect of the self-field within a ring.

\section{SIMULATION RESULTS}

The diode is modeled using an electrostatic 2D field-map imported into GPT to calculate the particle trajectories. The particles are emitted from the cathode with a radius of $0.25 \mathrm{~mm}$ and an initial emittance of $0.12 \pi \mathrm{mm}$ mrad. In the simulation the particles are emitted in time: Particles are added as they emerge from the cathode. The simulations were carried out to determine the beam characteristics using the pulsed diode and to optimize the geometry.
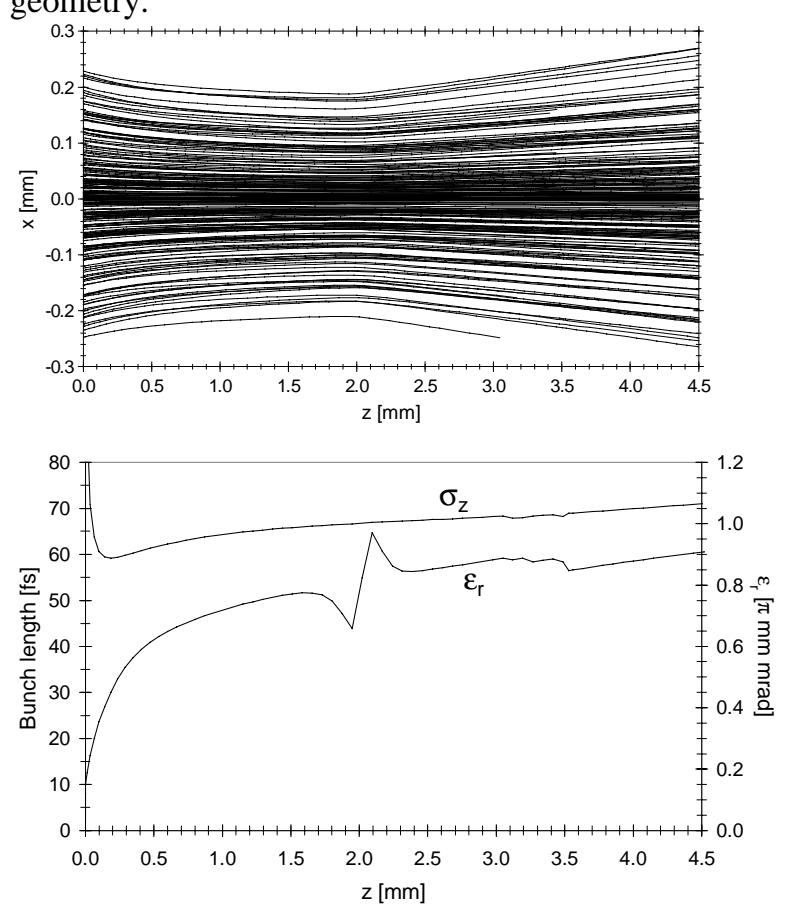

Figure 2: Particle trajectories in the diode and the evolution of the bunch-length and emittance. The beam is clipped slightly by the anode. 
Figure 2 shows the beam behavior in the diode. It can be seen that the flow is laminar, but $6 \%$ of the bunch is clipped at the anode. Emittance growth mainly occurs in the first $500 \mu \mathrm{m}$, when the beam energy is less than $0.5 \mathrm{MeV}$. The exit emittance is determined by the spacecharge forces and the normalized transverse emittance at the anode is $0.85 \pi \mathrm{mm}$ mrad with a RMS bunch length of $70 \mathrm{fs}$. These values meet the set criteria, but they have to be maintained in the second accelerator.

The curved cathode produces a waist in the beam, thereby eliminating the need for external focusing. The radius of the curvature has been optimized. A small curvature radius increases the bunch-length and the emittance, while a large radius causes clipping of the beam at the anode. We have chosen to use a cathode curvature radius of $2 \mathrm{~mm}$ because this greatly improves the beam qualities for a beam loss of only $6 \%$.

The initial beam radius has a significant effect on the bunch length and emittance. A small radius causes beam loss in the anode because the beam blows up due to spacecharge, while a large initial radius increases the bunch length and emittance.

\subsection{Radial laser profile}

The previous GPT simulations are performed with a uniform radial laser intensity function. According to Serafini [6] the best emittance results are obtained by clipping a laser beam with a gaussian radial intensity distribution at a radius specified by:

$$
R=\sqrt{\frac{2}{3}} \sigma_{r} \approx 0.8 \sigma_{r}
$$

where $\sigma_{\mathrm{r}}$ is the RMS radius of the gaussian distribution. To investigate the effect of a different radial laser profile, we varied the cut-off distance, relative to sigma, of a gaussian intensity profile while keeping the initial beam radius constant at $R=0.25 \mathrm{~mm}$. The transverse emittance has an optimum when the laser is truncated at $\sigma_{\mathrm{r}}=R$, as shown in Figure 3. The emittance is improved by $10 \%$.

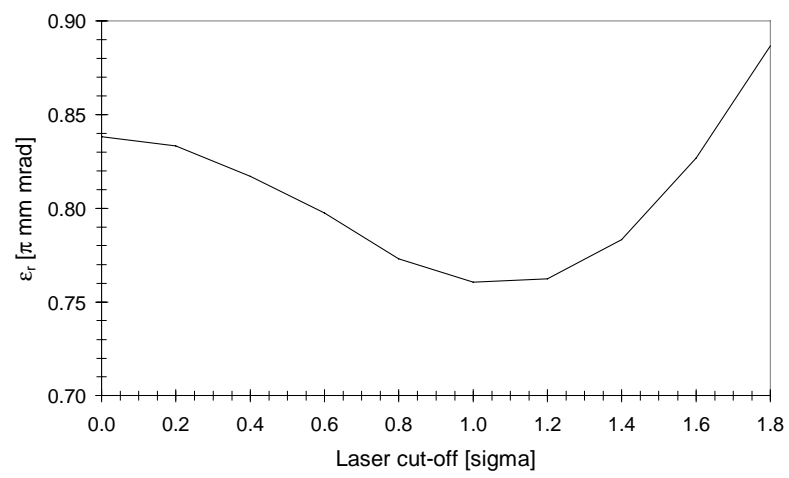

Figure 3: Transverse emittance as function of cut-off distance of a truncated gaussian radial laser intensity profile. The beam profile is varied from uniform (left) to almost a full gaussian (right).

\subsection{Bunch charge}

To better understand the beam dynamics in the diode, the effect of the charge in the bunch has been investigated. As shown in Figure 4, the bunch length grows linearly with the initial charge, with an offset of the laser pulse length. A high initial charge in the bunch increases the beam loss due to clipping at the anode aperture. The emittance at the exit of the diode grows with the bunch charge exiting the anode.

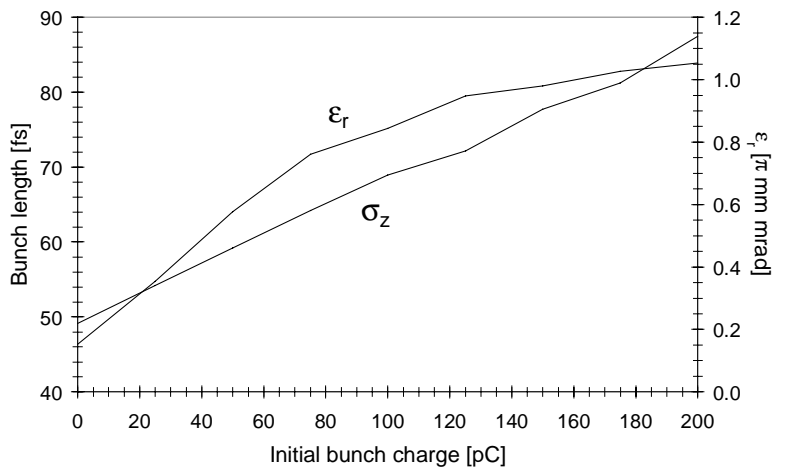

Figure 4: Effect of the initial bunch charge on the bunch length and emittance at the anode exit.

\section{CONCLUSION}

The beam parameters at the exit of the diode are very promising. We have obtained, in simulations, a bunch of $94 \mathrm{pC}$ with a bunch length of $70 \mathrm{fs}$. The transverse emittance is $0.85 \pi \mathrm{mm}$ mrad for a beam of $2 \mathrm{MeV}$. Currently we are designing an RF booster capable of maintaining these characteristics.

\section{ACKNOWLEDGEMENTS}

The authors gratefully thank Luca Serafini for his interest in this project and his stimulating comments.

\section{REFERENCES}

[1] K. Batchelor et al., Proc. 1998 Particle Accelerator Conf., Stockholm, Sweden (1998) pp. 791.

[2] S.B. van der Geer, M.J. de Loos, Proc. 1998 Particle Accel. Conf., Stockholm, Sweden (1998) pp. 1245.

[3] GPT User Manual, Pulsar Physics, De Bongerd 23, 3762 XA Soest, The Netherlands, http://www.pulsar.nl/gpt.

[4] J.H. Billen, L.M. Young, POISSON SUPERFISH, Los Alamos National Lab. Report LA-UR-96-1834.

[5] T.P. Wangler, Principles of RF Linear Accelerators, John Wiley, New York (1998), pp. 271.

[6] L. Serafini, Proc. Advanced Accelerator Concepts Workshop, Port Jefferson (NY) 1992, AIP Conf. Proc. 279 (1993) 645. 